\title{
A Clinical System Integration Methodology for Bio-Sensory Technology with Cloud Architecture
}

\author{
Abel Garai, Istvan Pentek, Attila Adamko, ${ }^{a}$ and Agnes Nemeth ${ }^{b}$
}

\begin{abstract}
Here, we outline the design, implementation, testing and evaluation phases of our bi-directional semantic and syntactic interoperability framework interconnecting traditional healthcare, industrial telemedicine and IoT wearable eHealth-domains. Specifically, our study demonstrates system interoperability among a hospital information system, an industrial telemedicine instrument and an eHealth smart wearable consumer electronic product through the Open Telemedicine Interoperability Hub (OTI-Hub) embedded in a hybrid Cloud architecture. The novelty of this study is the handling of Internet-ofThings smart healthcare devices and traditional healthcare devices through the same Cloud-based solution. This healthcare interoperability solution, service architecture and corresponding software engineering technique bridges technology barriers among the above-mentioned healthcare segments. Standard interoperability solutions exist and have already been described in related literature, but they are not applicable to the IoT healthcare devices and vice versa. Our study goes beyond isolated, individual interoperability solutions and seeks to bridge all major healthcare architecture frameworks including classical, telemedicine and eHealth IoT applications and appliances. This study presents the results of a two-year OTI-Hub Research Program. These experiments are manifestations of a trilateral cooperation among the University of Debrecen, Faculty of Informatics, the Semmelweis University 2nd Department of Paediatrics Pulmonology Division and an international hospital information system service provider.
\end{abstract}

Keywords: telemedicine, interoperability, bio-sensory monitoring, hybrid cloud, Hospital Information System, Health Level Seven, spirometry, Internet of Things

\footnotetext{
${ }^{a}$ Department of Information Technology, Faculty of Informatics, University of Debrecen, Kassai út 26, H-4028 Debrecen, Hungary, E-mail: \{garai.abel, pentek.istvan, adamko.attila\}@inf.unideb.hu

${ }^{b} 3$ Department of Pulmonology, 2nd Paediatrics Clinic, Semmelweis University, Tuzolto u. 7-9, H-1094 Budapest, Hungary E-mail: nemeth.agnes1@med.semmelweis-univ.hu
} 


\section{Introduction}

\section{$1.1 \quad$ Background}

Today, telemedicine interoperability technology is applied in aeronautics, maritime and astronautics. Feasible telehealth information technology is a critical success factor for future planetary exploration programs as well: The ISS [International Space Station] and future planetary exploration-class missions (e.g., to Mars) will require the incorporation of up-to-date telemedicine concepts and technology, subject to the resource restraints and operational realities of space medicine [1]. Even though in this study we present results of land-based healthcare systems interoperability research, the conclusions reached here are quite general.

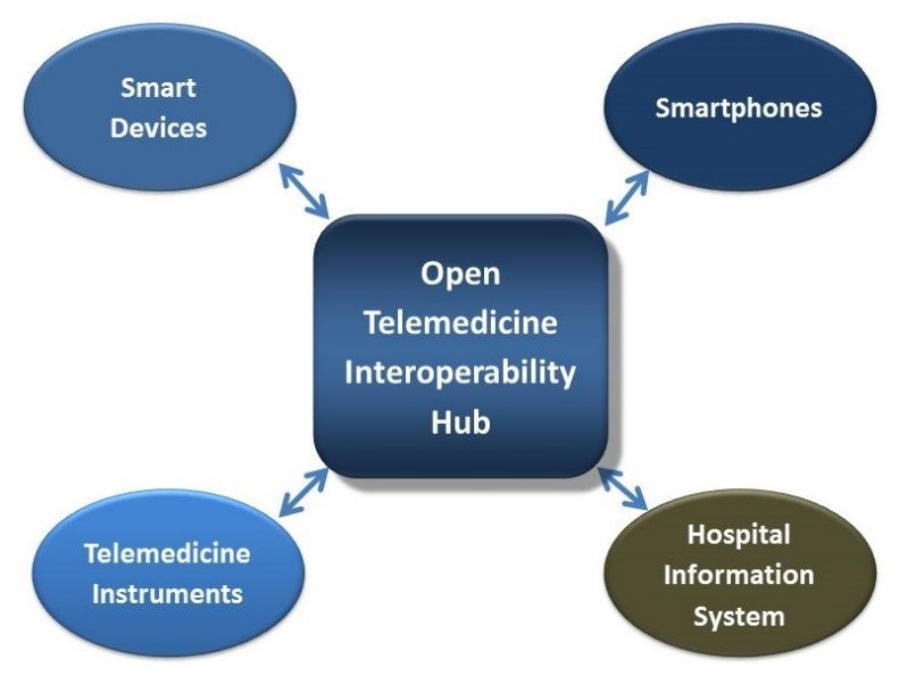

Figure 1: OTI-Hub Data-Link Diagram Schematics

Our research project commenced in 2015. We asked ourselves and tested whether there exists an information technology solution that allows interoperation among any biosensor enabled eHealth smart devices and any hospital information systems. Three technology sub-domains are specified in our program; namely eHealth smart device technology, telemedicine instruments technology and healthcare information system technology. If the hypothesis is proven, then in theory, there is no unresolvable technological obstacle preventing the interconnection of all eHealth smart devices, telemedicine instruments and hospital information systems. Furthermore, an extension of this would be in theory that all eHealth smart devices (eHSD), telemedicine instruments (TI) and healthcare information systems (HIS) could interoperate on a global scale. Here, current international healthcare interoperability standards and nomenclatures are taken into account as well. The crucial question for this research program is: can we build a (global) healthcare 
ecosystem based on the Internet of Things framework? Our study is a synthesis of traditional healthcare information system architecture, the telemedicine instrument landscape, and mobile wearable bio-sensory technology. An integration solution, Open Telemedicine Interoperability Hub (OTI-Hub, Figure 1), relying on a stateof-the-art international healthcare interoperability standard, has been developed, put into operationimplemented and functionally evaluated by quality assurance procedures.

\subsection{Research goal}

The issue of interoperability among clinical systems, telemedicine instruments and eHealth smart device technology is currently unresolved. There is a need for the seamless technical and methodical interoperability among these three different healthcare domains because:

1. The emerging sensor-based smart devices collect and transmit large amount of data,

2. Sensor-based smart devices collect and transmit semi-continuous data series,

3. Clinical information systems transmit and store static data with reference to patient records,

4. The data representation, structure, method and rationale are different in hospital information systems than in eHealth smart devices.

Overall, the technical solution, bridging the usual clinical information systems and the eHealth smart devices, remains still a missing link.

This study attempts to establish an empirical basis for a universal between clinical systems and Internet of Things (IoT). Our purpose is to place the interoperability (represented by the OTI-Hub) in the center, and facilitate communication with all the above-mentioned domains (HIS, TI, eHSD) over the Cloud-based OTIHub. The key goal of the study is a design concept that meets this scientific and methodological need, if possible. Below we demonstrate the theoretical basis for this interoperability design concept. Based on it, the appropriate technical architectural landscape has been developed. Using this architectural concept the system components have been identified, defined and developed. The interoperability among the interconnected system components developed by us has been tested, documented and evaluated in the study. The technical evaluation was followed by clinical validation (Semmelweis University 2nd Department of Paediatrics Pulmonology Division) in order to ensure that the technical and clinical information obtained are both interpretable. This study intends to apply healthcare supply-chain interoperability factors beyond strictly defined information technology considerations, including applicability in everyday clinical workflows. 


\subsection{From a Healthcare Practitioners Point of View}

Diabetes remains the number one global endemic disease. Insulin pumps developed for diabetes patients with sensors implanted under the skin require stable data-circulation. This solution supplemented with a Cloud-based interface and data-processing software transfers the actual value of the blood sugar level to the designated diabetes center via telecare and telemedicine software systems. The patients actual blood sugar information is displayed directly at the diabetes center, and the blood sugar timelines and periods are monitored and customized according to the patient's ailments.

The other significant group of endemic diseases is chronic asthma. Mobile telemedicine device developed for asthmatic patients is used by the diseased at his home and the results are automatically uploaded to the asthma clinical center through the Internet. The specialist analyzes the results during the patient's next visit or conducts a remote medical intervention, where necessary. The medical professional also evaluates the spirometry test results based on system standards.

Similar procedures are used in ECG tests and in the monitoring the the blood pressure monitoring of patients with cardiovascular disease.

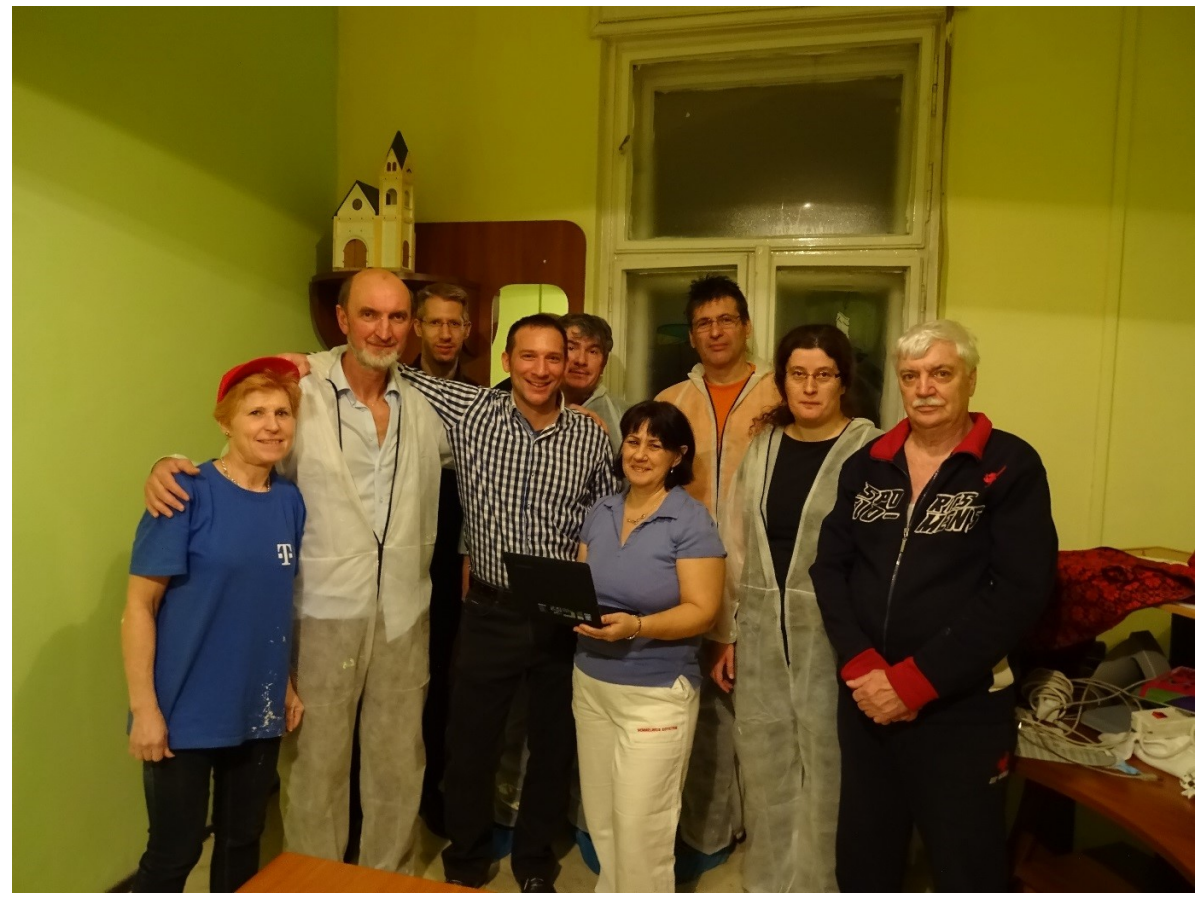

Figure 2: Semmelweis University 2nd Paediatric Clinic Department of Pulmonology after the successful interconnection of an experimental tablet through dedicated WLan access to hospital information system 


\subsection{Research partnership}

Finally, within the research project, the University of Debrecen, Faculty of Informatics, Department of Information Technology provided a good basis for ICTrelated tasks. Secondly, the Semmelweis University 2nd Department of Paediatrics Pulmonology Division provided the medical background for the project (Fig. 2). Thirdly, an IT technology service provider at a healthcare competence center delivered the test system to the hospital in order to perform a series of experiments. The University of Debrecen and the Semmelweis University Clinic provide nearly ten percent of domestic healthcare coverage.

\subsection{Experimental Laboratory Hospital Information System}

Interoperability use cases were tested on a test-acceptance instance of the chosen hospital information system (MedSol). This hospital information system operates in sixty hospitals over Europe and serves forty thousand users. There are other international healthcare software providers on the market as well, including SAP Healthcare Solutions, IBM HospiLogix, Avicenna; Siemens Soarian Clinicals, GE Centricity and Oracle Healthcare Management Platform. However, the University Clinics of Debrecen and the Clinics of Semmelweis University have the necessary software licenses to run the MedSol system. The limited budget of the project did not allow us to test the OTI-Hub on other manufacturer's hospital information systems. Therefore, testing the HIS interoperability of different manufacturers through the OTI-Hub fell outside of the scope of our study.

\subsection{Spirometry}

The PDD-301/shm medical spirometer installed in out system represents the telemedicine instrument landscape in the research project. This medical telemedicine device is connected through the OTI-Hub, converted into Health Level Seven (HL7 [2, 3])-based interface files and transmitted to the hospital information test system.

A heart-rate monitoring smart wearable bracelet forms part of the eHealth smart device technology domain.

During the study the industrial telemedicine instrument interconnection was made at the Semmelweis University 2nd Department of Paediatrics Pulmonology Division, and the PDD-301/shm spirometer is connected to the clinical information system there (MedSol and eMedSol; figures 3 and 4). This system is accessed via tablets through local clinical WLAN by the medical staff. Our study well explain how the mobile spirometer and the also mobile clinical information system GUI cooperate with each other. This will be in effect a mobile telemedicine deployment simulation, like healthcare solutions for remote, sparsely populated regions like some cities in Norway, Sweden and Canada. Here, our study strictly applies the international HL7 healthcare interoperability standard. 


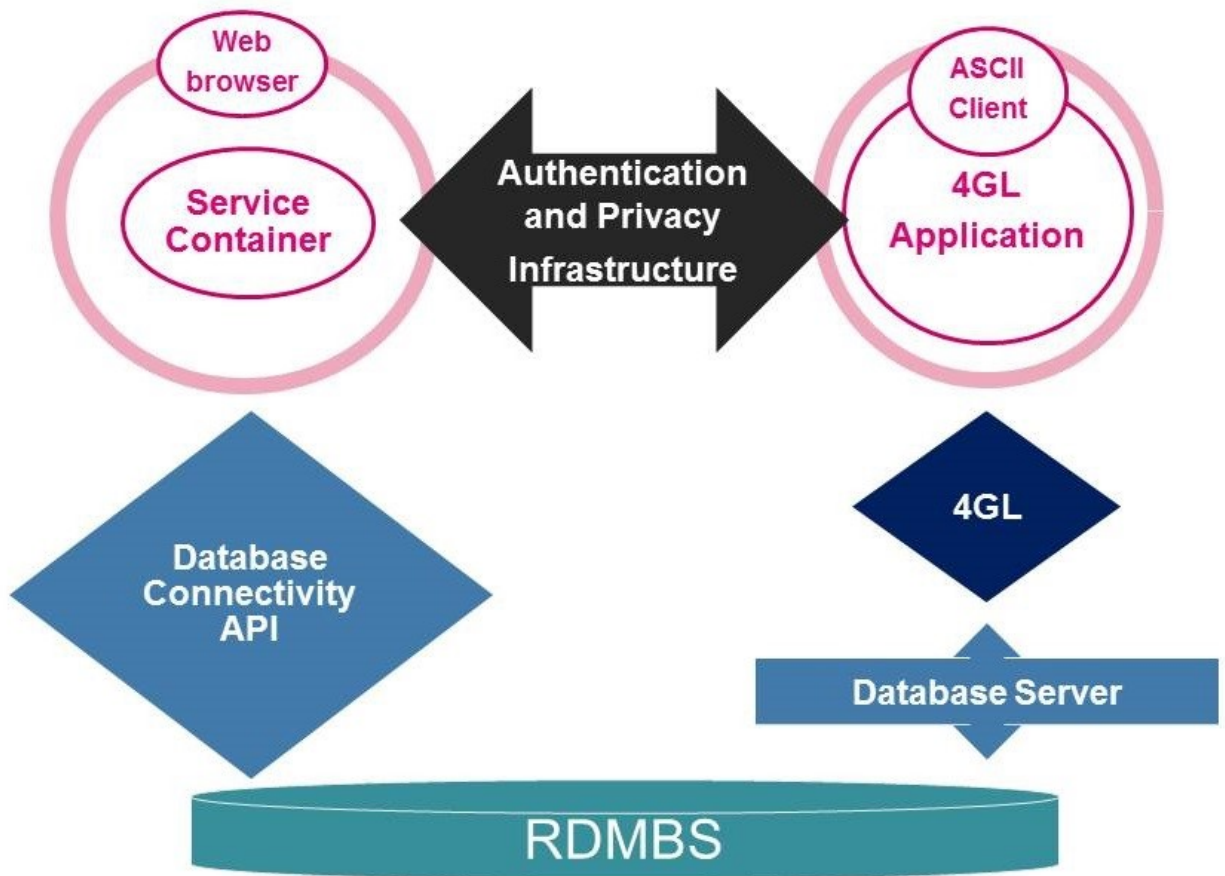

Figure 3: Typical Hospital Information System Schematic Architecture

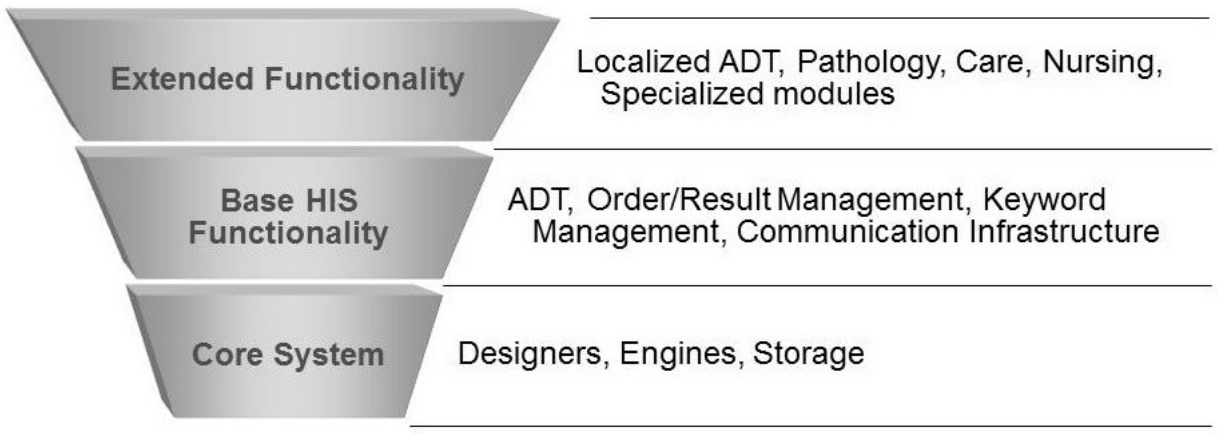

Figure 4: Modular-Hierarchical Hospital Information System Concept

\subsection{Influential industry megatrends}

Present IoT trends suggest that within a decade, billions of smart devices will be communicating nonstop with each other: Cisco estimates that by 2020, 50 billion devices and objects will be connected to the Internet [4]. As for the eHealth smart 
device market, penetration should mean billions of new users will be included within the near future [4], so the healthcare information gathered so far is quite valuable. Industrial Big Data [5] analytical capabilities combined with incoming personal health data volumes open new horizons in personal and community healthcare forecasting including international epidemic control.

Global cloud infrastructure capabilities has reached its maturity over the past decade, and now they are ready to handle bulk sensory data flow. This allows us to develop Cloud-based interoperability solutions tuned for mass sensory-data handling, like the Open Telemedicine Interoperability Hub.

Cloud architectures are classified into private, public and hybrid clouds (community cloud is excluded in this study due to reliability and availability concerns). The private Cloud offers feasible technical solution for sensitive personal patient data; and the public Cloud delivers the necessary scalability.

Related Works are evaluated in Section 2. Section 3 determines the suitable syntactic healthcare systems interoperability standard and nomenclature. The clinical systems interoperability improvement proposal and standard recommendation is described in Section 4. The recommended service architecture topology, our design concept and deployed software technology is defined in Section 5. Section 6 stipulates the impact evaluation and technical research report including threats to validity. In Section 7, we discuss future work. Finally, in Section 8 we summarize our conclusions and views.

\section{Related Works}

A recent study has created a general software platform for clinical systems integration of telemedicine instruments [6]. However, these studies cited remain on a theoretical basis. Nevertheless, the available studies focus only on linking the telemedicine instruments with the mobile wearable body-sensory appliances.

All the cited literature treat the HIS and the IoT eHealth domain as two characteristically separated ecosystems. The relevant literature generally places the HIS at the center of the medical information technology landscape [7]. The studies in the field of interoperability focus either on the HIS-TI or on HIS-IoT interoperability. No comprehensive research has been found on handling the inter-domain (HIS-TI-IoT) interoperability.

\subsection{Telemedicine instruments interoperability}

Telemedicine instrument (TI)-hospital information system (HIS) interoperability has already been thoroughly studied and summarized in the relevant scientific literature $[8,9,10]$. International standards have been implemented regarding syntactic-level interoperability and healthcare data exchange (HL7, GDT, etc.). Process interoperability has also been thoroughly analyzed and scientists already have delivered proposals to solve the current gaps [11, 12]. However, regarding the TI-HIS interoperability, there is still plenty room for research in the area of non- 
orthodox data exchange integration, like multilateral videoconferencing and diverse patient-information sharing (including, but not limited to transmission, capture and the interpretation of unstructured text and images) $[13,14]$.

\subsection{Microcontroller-level interoperability}

In the available literature microcontroller- and protocol-level generally applicable interoperability solutions are provided, like Sensor Hub and Sensor Hub Cloud Servers. Perera et al [15] describe their low-level interoperability solution with linkage to cloud-server processing. Lengyel et al present their general sensor hub framework [16]. Shibuta and Iwata demonstrates data-driven sensor hub architecture [17]. Patel and Sola exhibits a wireless sensor hub communicating through WiFi protocols [18]. These solutions all provide a partial solution to the healthcare interoperability problem. The results presented in this paper focus on Cloud-based syntactic and semantic interoperability across the telemedicine, IoT eHealth and classical healthcare domains. The current and cited Sensor Hub solutions do not cover the entire topic of this study, but provide a particular solution to the problem.

\subsection{Consumer electronics and IoT eHealth interoperability}

Kumar et al present an integration architecture suitable for IoT consumer electronics transmitting continuous data signals [19]. This study is a milestone, but it covers only a limited part of the problem of the actual research. Notwithstanding that for the Internet-of-Things (IoT) appliance-HIS interoperability has already been studied in the relevant international literature, these focus strictly on the given topic but unfortunately they do not offer a coherent architectural or a single standard solution [20, 21].

\subsection{Regional healthcare interoperability}

Barbarito et al describe the comprehensive regional healthcare information system implemented in the Lombardy region in Italy [22]. This system integrates the healthcare services for ten million citizens, 150000 health- and social care workers, 7800 general practitioners, 2600 pharmacies, 35 public hospitals, 15 local healthcare units and over 2500 private healthcare organizations. This project is a noteworthy landmark for general healthcare interoperability in practice. However, IoT eHealth device integration is not handled in this implementation project, and it is applied stand-alone classic servers instead of cloud technology.

\subsection{Leading interoperability standards}

The key healthcare electronic communication standards are the international Health Level Seven (HL7) and the German xDT standard family [23]. The xDT standard family consists of the GDT (Gertedatentransfer, instrument data transfer), BDT (Behandlungsdatentransfer, treatment data transfer) and LDT (Labor- 
datentransfer, laboratory data transfer) data exchange format families. There are healthcare standardization organs like the USA-based National Council for Prescription Drug Programs (NCPDP, [24] and the also USA-based Organization for the Advancement of Structured Information Standards (OASIS, [25]). The Cloud Application Management for Platforms (CAMP, Standardizing cloud Platform-asa-Service Application Programming Interface [26]) is a standardization initiative of the OASIS entitiy.

\section{Applied Syntactic Healthcare Systems Interop- erability Standard}

The HL7 healthcare interoperability de facto international standard and the widely recognized SNOMED-CT [27] nomenclature have been chosen as the primary conditions for this study. The conceptual logic of the OTI-Hub system relies on the basis of the above mentioned standard and nomenclature. In theory, the vast majority of HIS and medical instruments are HL7-enabled [28]. However, in reality the HL7 standard itself has different, not fully compatible sub-versions (HL7 v2.x, HL7 v3). Notwithstanding that the latest HL7 v3 standard covers almost all internationally applicable medical information areas instead of the previous HL7 v2.x version [29], the HL7 v2.x is the de facto international interoperability standard for clinical information systems according to industry consultants. This is why we applied the HL7 v2.3 standard for the clinical spirometer-healthcare information system interconnection.

The HL7 v2 family consists of comma separated files (CSVs), while the HL7 v3 group has an XML structure. Transaction manager software products (like IBM Websphere and the MQ-Series) can handle both CSV and XML files successfully, truncate them when needed and forward the designated CSV or XML part to the recipient system or database table. Both CSV and XML files are applied in other heterogenous system landscapes, like global card transaction processing systems (e.g. VISA) and in billing systems (e.g. German Telekom Landline Billing).

This step (the application of the HL7 v2 instead of HL7 v3) provides a significant constraint. The recent HL7 v3 versions deliver a broad interoperability capability. However, our team was told by an industry consultant that the vast majority of the hospital information system deployment within Central- and South-Europe apply the older HL7 v2 version. This fact should have been taken into account in our study. The OTI-Hub itself is both HL7 v2 and v3 capable, but in the course of the testing the de facto HIS limitations influenced our project plan. The parametrized HL7 v3 output from the OTI-Hub is shown on Figure 5. It represents a fasting blood sugar (FSB) measurement after the patient had not eaten for at least eight hours. Usually the FSB is the first test to check for prediabetes and diabetes. The above-mentioned XML defines the accepted low and high values with a specified unit, but these are the extreme values. In this case, the specified unit is milligrams per deciliter. The measurement has an explicit value of 182 milligrams per deciliter. With this measurement, the specialists could diagnose 
diabetes, because the measured value of over 105 milligrams per deciliter hit the defined maximum value.

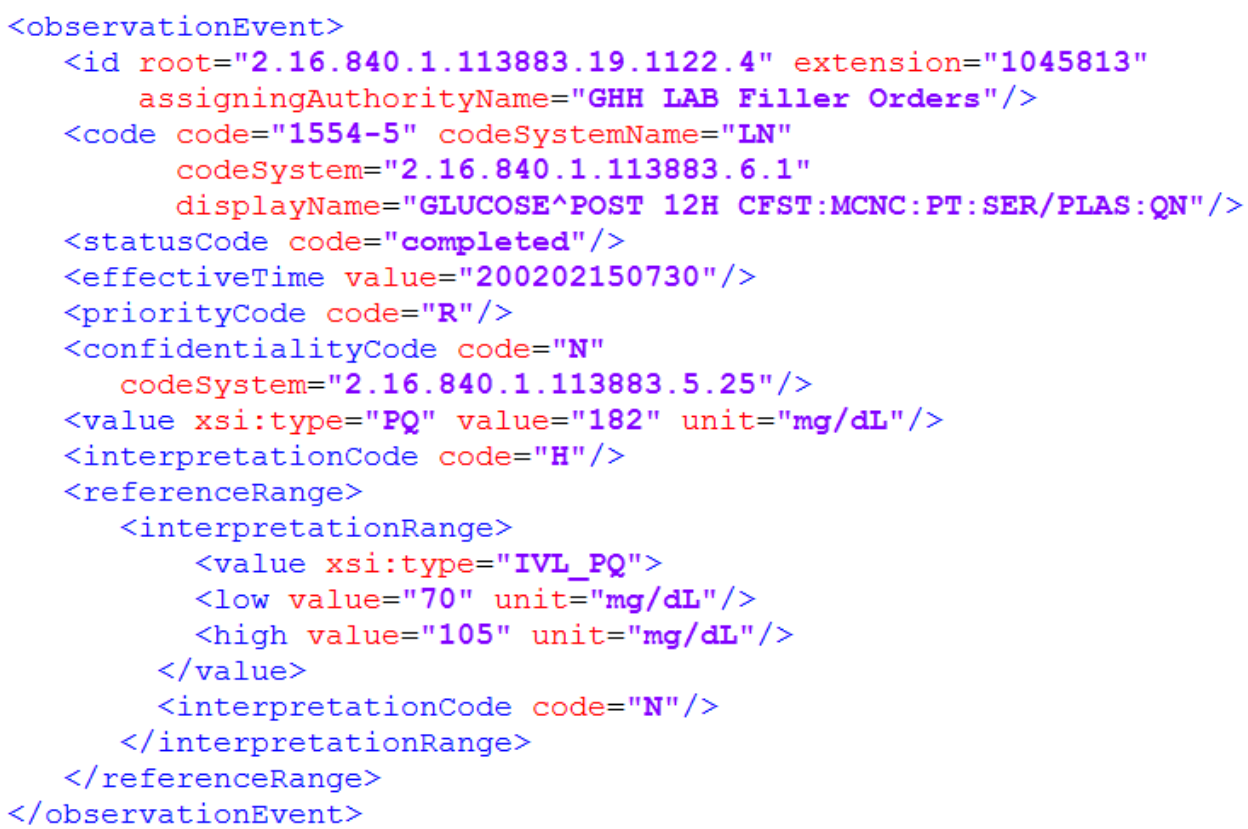

Figure 5: Parameterized HL7 v3 standard output from the OTI-Hub

The integration of the eHealth smart device technology within the classical medical system landscape is also necessary for the overall enhancement of the healthcare supply chain [30]. It establishes a link for traceable information exchange among conventional medical systems, industrial telemedicine instruments, eHealth smart devices and adaptive healthcare-services [31, 32]. The OTI-Hub system relies upon the international HL7 standard and provides bi-directional interoperability among a HIS, an eHSD and a TI $[33,34]$. The OTI-Hub is embedded in a Cloud Architecture. This architecture brings significant benefits, but also takes into account patient data privacy issues.

\section{Clinical System Interoperability Improvement Proposal and Standard Recommendation}

Our goal is to create a flexible telemedicine interoperability hub that will extend the options of conventional hospital systems. To achieve this goal, industry-wide accepted technologies have been applied [35, 36]. Also, the software development environment for the hub has been selected and made concrete (the selected technologies are available on Microsoft stack, and the OTI-Hub system has been im- 
plemented with Microsoft stack and other open source technologies [37]).
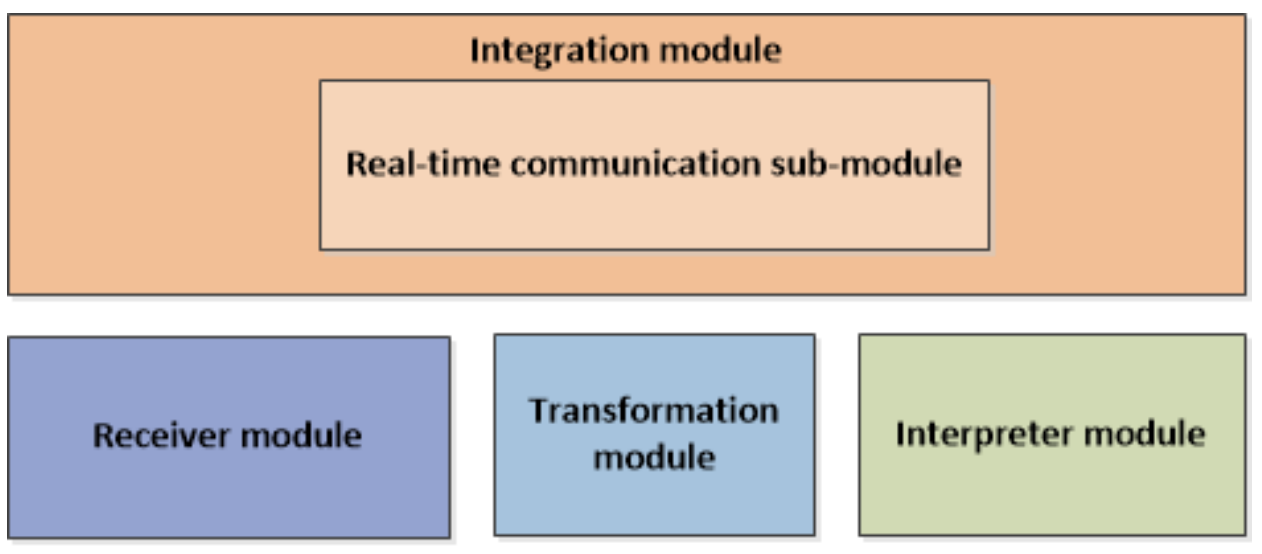

\section{Storage module}

Figure 6: OTI-Hub Service Architecture

The following technologies were applied within the OTI-Hub (Figure 6) and in the modules:

The receiver module handles the received measurement data through http Web request. This module uses a Web API library and associated markup language (represented by Microsoft Web API, JSON and XML in the study). This module applies an open authentication protocol (OAuth in this study) to authorize and authenticate the users and devices. The REST principles also need to be adapted, where possible $[38,39]$.

The transformation module operates on the data collected by the receiver module and the main task of this module is to transform the date to the chosen interoperability standard format (HL7 and C\#-based Windows service in the study). This module is of course a critical part of the system. As eHSD manufacturers specify different output formats, the transformation to a unified format is a cornerstone for interoperability. The key to the OTI-Hubs general expansibility is an incoming and outgoing data format conversion. At the moment the transformation module is the OTI-Hubs internal component. An independent transformation module should be planned, built and deployed later, so as to make the Hub generally applicable.

The data storage module is responsible for building a data warehouse from the data that was collected and transferred. The OTI-hub uses the storage part of Apache Hadoop, known as the Hadoop Distributed File System (HDFS), to store the measurement data. It is a file based storage solution, where the Hadoop splits the files into very large blocks and distributes them across the nodes in the cluster. 
The interpreter module works with the data warehouse module and its main task is to interpret the data that was collected and aggregated. The OTI-hub uses the Apache Hadoop software library to evaluate the measurement data. Apache Hadoop utilizes the MapReduce programming model. It transfers package codes into cluster nodes to process the data in parallel. This approach takes advantage of data and file locality, every node manipulating the data and files they have access to.

Here, the key module is the Hubs integration module. This module sends export data features from the telemedicine hub to external systems like hospital information systems. This module uses REST API endpoints to transport data to external systems; and it applies the technologies summarized in the receiver module (Microsoft Web API, OAuth, JSON and XML in the study [40]).

The real-time Web communication sub-module library operates in order to maintain an open socket between the Hub and the devices (SignalR in our study). This module is critical when a device is recording data frequently and the received data have to be readily availabe on the Hub. In this case, when lower priority measurement data are not needed immediately on the Hub, this module can be excluded from the process chain. The socket allows one to use a channel between the Hub and the device without reconnection and re-authentication. The only significant latency is network latency in this case. The OTI-Hub is suitable for a full duplex channel, so the data can be transmitted and received in both directions.

The Hub is embedded in a Hybrid Cloud Architecture. The Hub component, responsible for the patient-related master data, is embedded in a private cloud architecture (German Telekom Private Cloud in our study). The Hub-components, responsible for the interoperability logic and routing, are implanted in a public Cloud (the Google Cloud Platform in our study).

Here, the solution presented here is an open architectural solution providing the basis for healthcare interoperability. It is open for the future expansion and inclusion of new specific technologies. Based on the Hub's internal structure, it can be adjusted to varying incoming formats. By reparametrizing the Hub, it can process other custom protocols as well (e.g. bio-sensory dataflows forwarded by cell-phones).

\section{The Recommended Service Architecture Topol- ogy and Software Technology}

\subsection{The Service Architecture Topology}

Here, our aim is to set up a service architecture through the OTI-Hub that will enable bi-directional syntactic interoperability among HIS, TI and eHSD. Therefore, we established a cloud-based system landscape (Figure 7) based on the OTI-Hub and the HL7 interoperability standard.

The TI is interconnected with a personal computer (PC) via a USB. The factory client program runs on this PC. This is interconnected by a Wide Area Network 


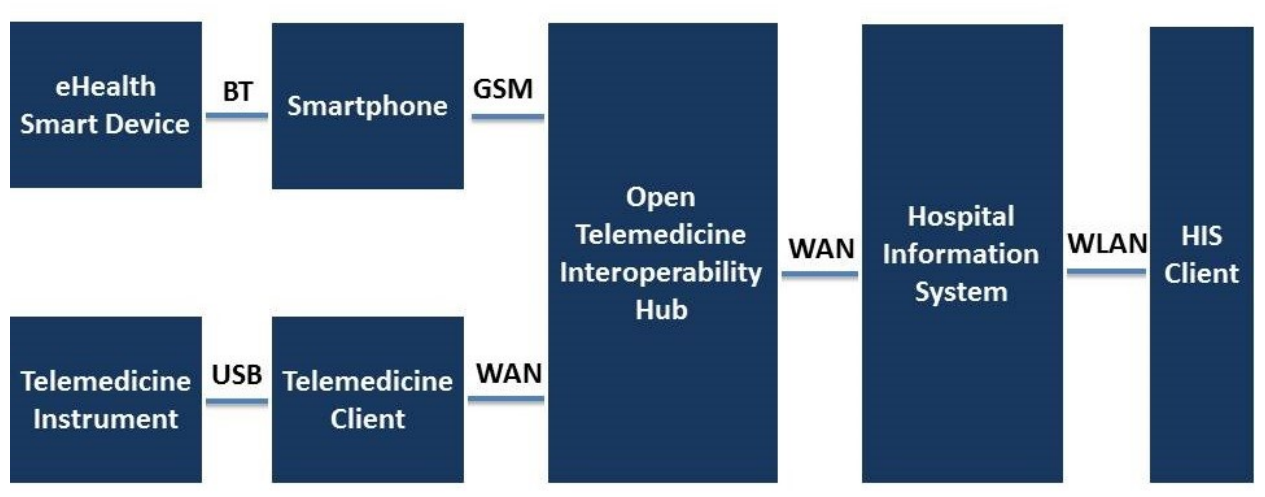

Figure 7: Telemedicine and eHealth Systems Interoperability Landscape with OTIHub

Internet connection to the cloud-based OTI-Hub. The eHSD communicates via a Bluetooth connection to the smartphone, which in turn runs the OTI-Hub App. This App, developed by our research team may be downloaded and installed by the Windows App Store to this device. The smartphone connects via a GSM or W-Lan internet connection to the OTI-Hub. The OTI-Hub is connected via a WAN Internet-connection to the HIS. The prototype tablets are connected by a dedicated internal W-Lan connection to the HIS, and the HIS online Graphical User Interface (GUI) runs in the tablet's browser.

The novelty of this architecture is that it integrates all the conventional healthcare information system architecture, the standard telemedicine environment and the eHSD technology.

The cloud-based Healthcare Information System (HIS) we applied, called eMedsol currently operates in sixty hospitals and serves forty thousand users in Hungary, Romania, the Czech Republic and Bosnia-Herzegovina. The eMedSol system is equipped with a UNIX-based (Linux, AIX HP-UX, SCO) WebSphere Application Server (V5, V6) that is connected to Oracle (10gR2) and Progress (V10 OpenEdge) relational database management systems (RDBMS). Each installation (for a clinical institution with fewer than three hundred beds) is equipped with a primary and a secondary virtual server, two quad-CPUs and 16 GB Memory. The OTI-Hub is embedded in a Google public Cloud (Google Cloud Platform), and sensitive patient personal data are stored in the Mnchen-based Open Telekom Cloud datacenter to ensure that patient data remain within the EU.

The service architecture shown in Figure 8 provides the methodological basis for the kind of IT solution (e.g. OTI-Hub) that provides interoperability among the conventional healthcare IT components, telemedicine appliances systems and eHSD device technology. 


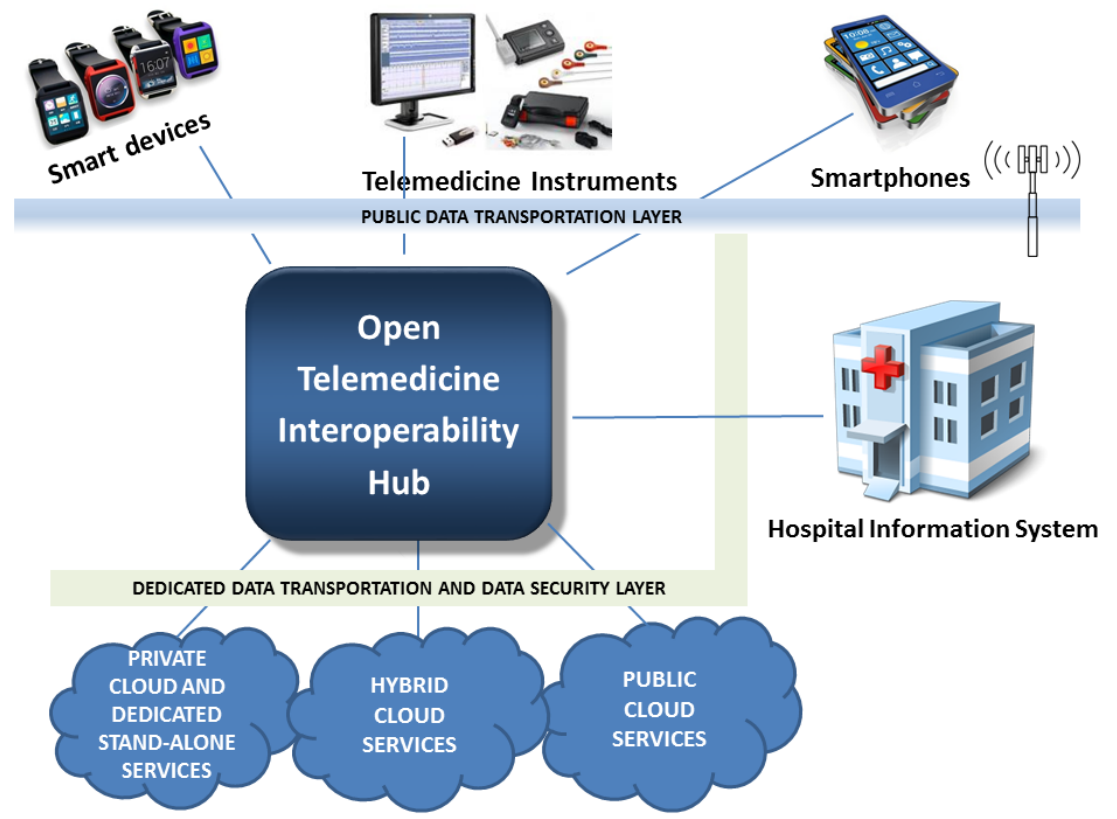

Figure 8: Our Open Telemedicine Interoperability Hub (OTI-Hub)

\subsection{Software Technology}

The concept of clinical systems interoperability goes far beyond simple data exchange: it constitutes interoperability at the technical, semantical and process levels. In an empirical model of the study the OSI model (ISO/IEC 7498-1:1994 [41]) is mapped against these interoperability levels, hence these three interoperability modalities can also be interpreted at the corresponding IT abstraction layer. In our study we focus on both technical and semantic interoperability. Among the technical interoperability modalities, instead of the TCP/IP, we chose the file-based interface connection, since this option offered significantly more flexibility during our study $[42,43,44]$. The clinical spirometer PDD-301/shm was linked over a USB connection to the corresponding spirometry desktop program provided by the manufacturer.

The following instruments were selected and allocated to the study program: the spirometer PDD-301/shm as a clinical telemedicine instrument, Microsoft Band I and Microsoft Band II smart wristbands as eHealth sensory devices, a Nokia Lumia 930 smartphone, the Windows 10 Mobile operating system, the Dell Latitude E6520 (Windows 10, 32-bit operating system, i5-2520M chipset, 4 GB RAM and 256 GB HDD) primary laptop, a Dell Latitude E6220 (Windows 764 bit operating system, i5-2520M chipset, 4 GB RAM, 128 GB SDD) secondary laptop, three Lenovo MIIX 300-10IBY tablets and an ACER SWITCH SW3-013-12CD tablet. 
Each tablet was equipped with a 10,1 display (WXGA and HD IPS), 2 GB memory, 64 GB internal storage and Windows 10 operating system. All the laptops and tablets were compatible with the 802.11g WLAN and Bluetooth 4.0 standards; and the spirometer is USB-enabled. The chosen smart wristbands were manufactured with built in- Bluetooth 4.0 communication chipsets. Each instrument of the lab equipment package was also individually tested prior to the experiments.

A specific private cloud was set up for our study. This ran on a stand-alone x86-64 architecture equipped with an Intel i5 processor, 256GB SSD and 4 GB RAM. The operating system for the private Cloud was Red Hat Enterprise Linux 7.0 3.10.0-229, the virtualization was provided by VMware Workstation v6.5.0 and the relational database management system was supplied by MySQL v5.6. The cloud-based version of the hospital information test system ran in a commercial private cloud (Telekom Cloud). The Open Telemedicine Interoperability Hub data transmission module was embedded in a commercial public Cloud called Microsoft Azure.

The HIS ran on J2EE WebSphere Application server V6, which relied on Oracle RDBMS 10gR2 and Progress V10 OpenEdge RDBMS. Also, the HIS was hosted on the Unix operating system. Floating licenses were made available for accessing the online, cloud-based edition of the HIS via the experimental tablets. The Open Telemedicine Interoperability Hub development environment consisted of the Universal Windows Application Development Tools (1.4.1), Windows 10 Software Development Kit 10.0.25431.01 Update 3 and Microsoft .NET Framework Version 4.6.01038. The OTI-Hub internal database was developed using the SQL Server Data Tools 14.0.60519.0. The OTI-Hub App was developed using Visual Studio Tools for Universal Windows Apps 14.0.25527.01. The OTI-Hub middleware was embedded in Microsoft Azure Mobile Services Tools 1.4. Red Hat Enterprise Linux 7.0 3.10.0-229 provided the operating system for the private cloud set up specifically for the study.

The spirometry desktop program was installed on a standalone Dell Latitude E6520 laptop equipped with the Windows 10 operating system. The spirometer was calibrated by the manufacturer for the study. A forced vital capacity spirometry test was performed with a healthy individual. After the test results had been stored in the spirometry desktop software package, the HL7 v2.3.1 interface file was exported. This interface file was processed by the cloud-based OTI-Hub. The OTI-Hub appended the spirometry information with the earlier transformed cardio body-sensor information captured by the L18 Smart Bluetooth Wristband. The generated HL7 interface file was imported after parameterization into the factory acceptance test instance of the MedSol hospital information system. Both the imported spirometry and cardio test results were retrieved and displayed by the patient report query of the hospital information system.

The OTI-Hub is embedded in a hybrid-cloud. The OTI-Hub patient-datarelated components ran within the Private Cloud Infrastructure-as-a-Service environment. The other OTI-Hub components ran within the public cloud Platformas-a-Service environment. 


\section{Impact Evaluation and Technical Research Re- port}

The IT results were validated by the Department of Information Technology, University of Debrecen and by our Industry Partner. The clinical results were validated by the Semmelweis University 2nd Department of Paediatrics.

The spirometry (see Figure 9) and cardio sensory HL7 test result data transferred via the OTI-Hub was successfully imported, interpreted and presented in the target HIS. As expected, the spirometry and cardio test results were correctly reflected through the HISs patient result query. The cardio information in the HIS query displayed the values transformed by the OTI-Hub. The results told us that HL7-based health data interchange among different Information Technology architectures was fully feasible. The OTI-Hub successfully provided seamless interoperability among Android-, UNIX- and Windows-platforms. Both cloud and standalone architecture components were also effectively interconnected during our study.

The HL7-based information exchange among the healthcare information acceptance system, the dedicated standalone spirometry system (see Figure 10) and the OTI-Hub was stable and trackable (see Figure 11). The combination and compatibility of the different HL7 versions were mainly handled within the OTI-Hub logic. The conversion of the body-sensory smart device output data stream into meaningful HL7 interface information was a significant challenge. While the spirometry output interface file was sent and processed by the OTI-Hub correctly, healthcare smart-device manufacturers unfortunately do not provide well-specified output format in most cases. These smart device output formats are typically readable for humans, but not precise enough for automatic processing [43].

In addition, the healthcare smart device output data streams are not ready to be processed by HIS unless they can be transformed into meaningful static values. However, our OTI-Hub successfully imported and interpolated the randomly selected healthcare smart devices (smart bracelet) data stream into interpretable HL7 values in this particular case. Naturally, this issue will require further investigation. As healthcare smart devices coming from different manufacturers use a very diverse output data-format and data emission frequency, there is room for overall standardization here to achieve a single format. It would be most desirable to find a solution for this issue. However, this topic goes far beyond the technical aspects: commercial interests, patent rights and other non-technical considerations influencing this area. From a technology perspective, sensor-Hub technologies, international biosensor-flow standards and best practices could deliver the desirable solution for this issue. However, finding a good solution for an industry-wide standardized primary bio-sensory data-format falls outside the scope of this study. To sum up, the actual results of the study meet the requirements, and provided significant useful lessons for us, as we suggested in the consclusions section (see Section $8)$. 


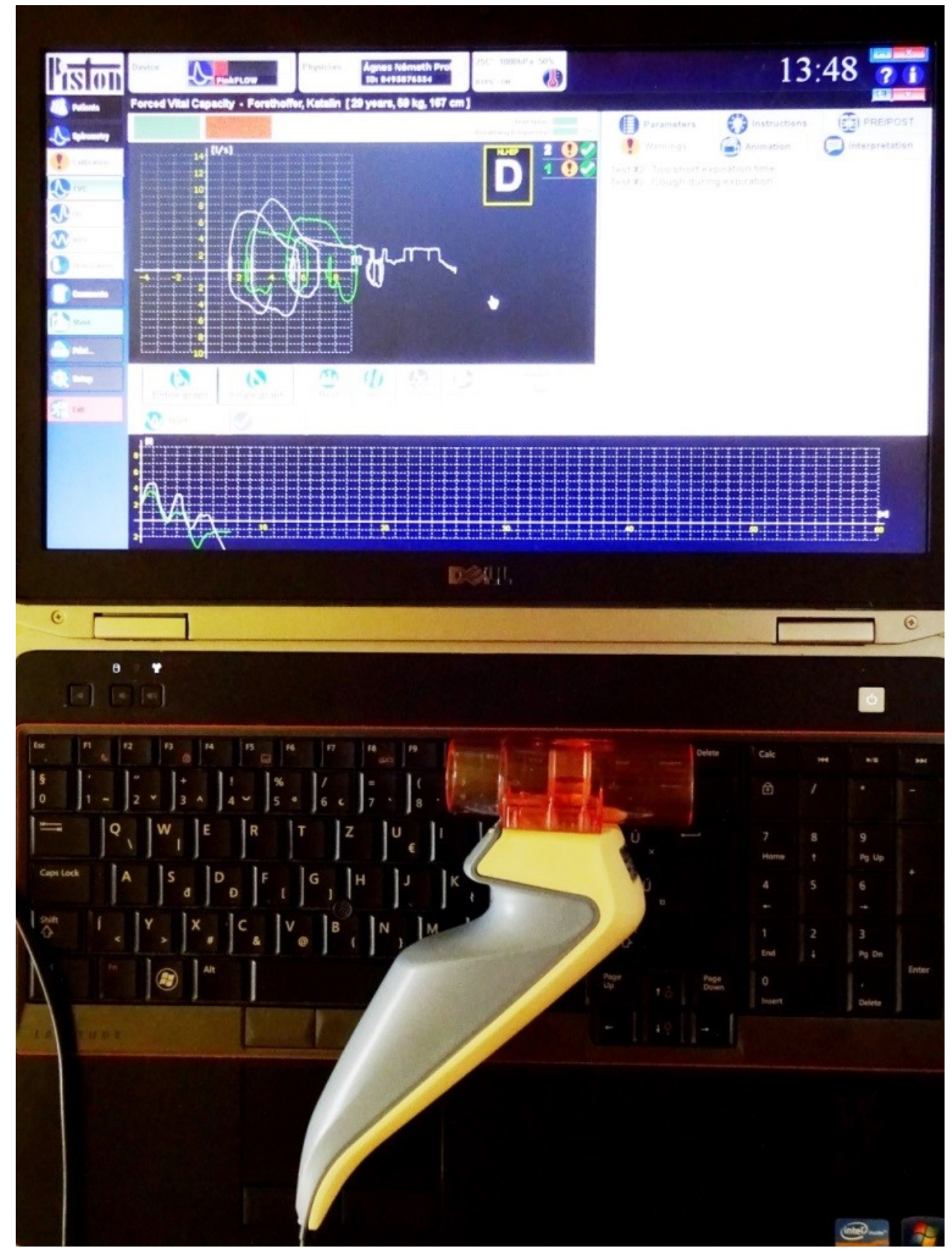

Figure 9: Data-interchange over our OTI-Hub with a clinical spirometer PDD$301 / \mathrm{shm}$ 


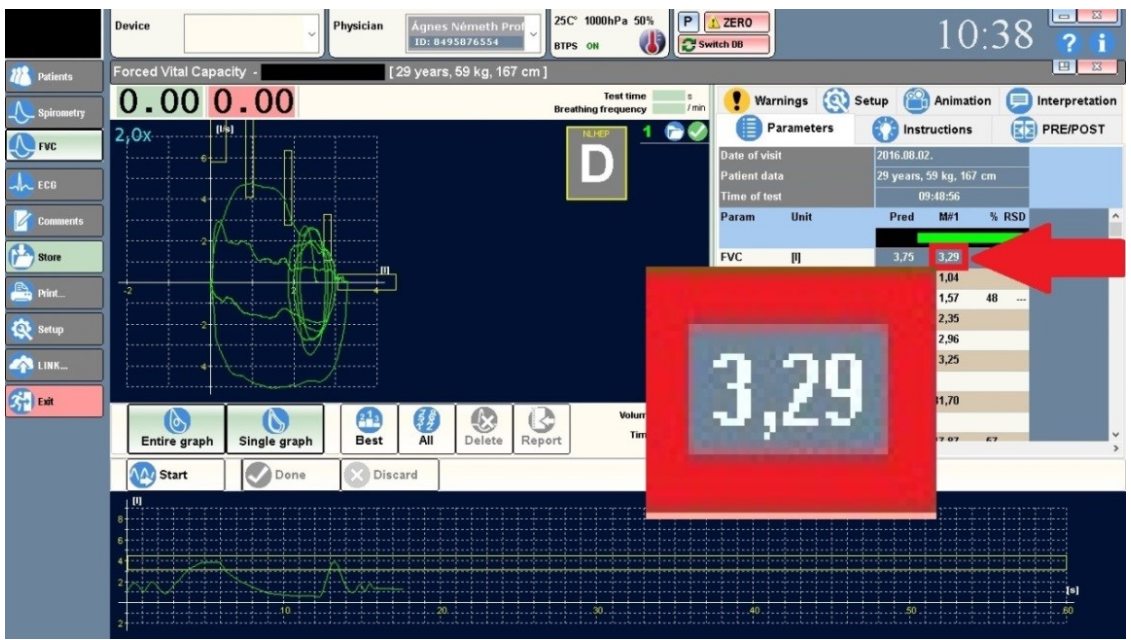

Figure 10: A Spirometry Clinical Test Result data-export to the OTI-Hub in HL7 v2.3.1 format

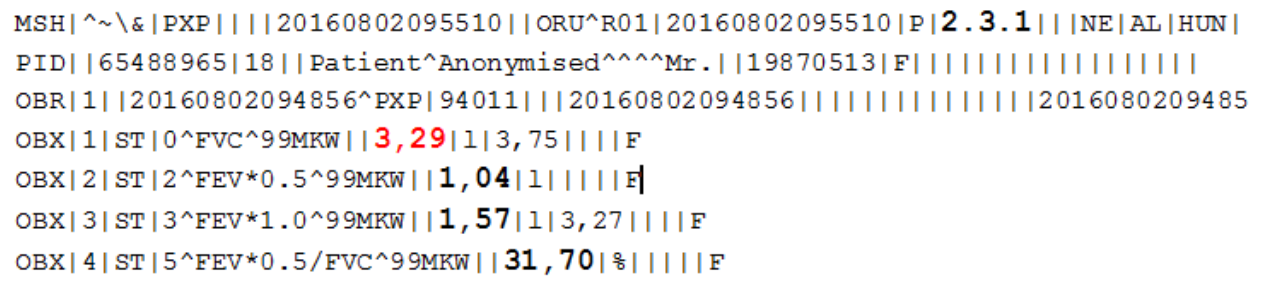

Figure 11: An extract from the Clinical Spirometer HL7 v2.3.1 Output File got via the OTI-Hub

\subsection{Threats to validity}

Our study did not analyze the data-flow in the backward direction, e.g. when data originating from a conventional HIS are sent to a healthcare smart app running on smartphone using the OTI-Hub. This backward healthcare information flow should be examined in a later study. Here, ours applied file-based interface connectivity. The data package based interconnection may react differently under real-life conditions, and it may have its advantages (speed) and disadvantages (blocked communication in the case of a broken link in the dataflow-chain). The commercially available eHSDs do not meet healthcare standards; therefore the intercepted data may not be clinically as precise as it is required (see Figure 12 and Figure 13).

Even global cloud providers have different regional datacenters and the results in geographically different levels of service. This will limit the performance of the OTI-Hub at the international level. 


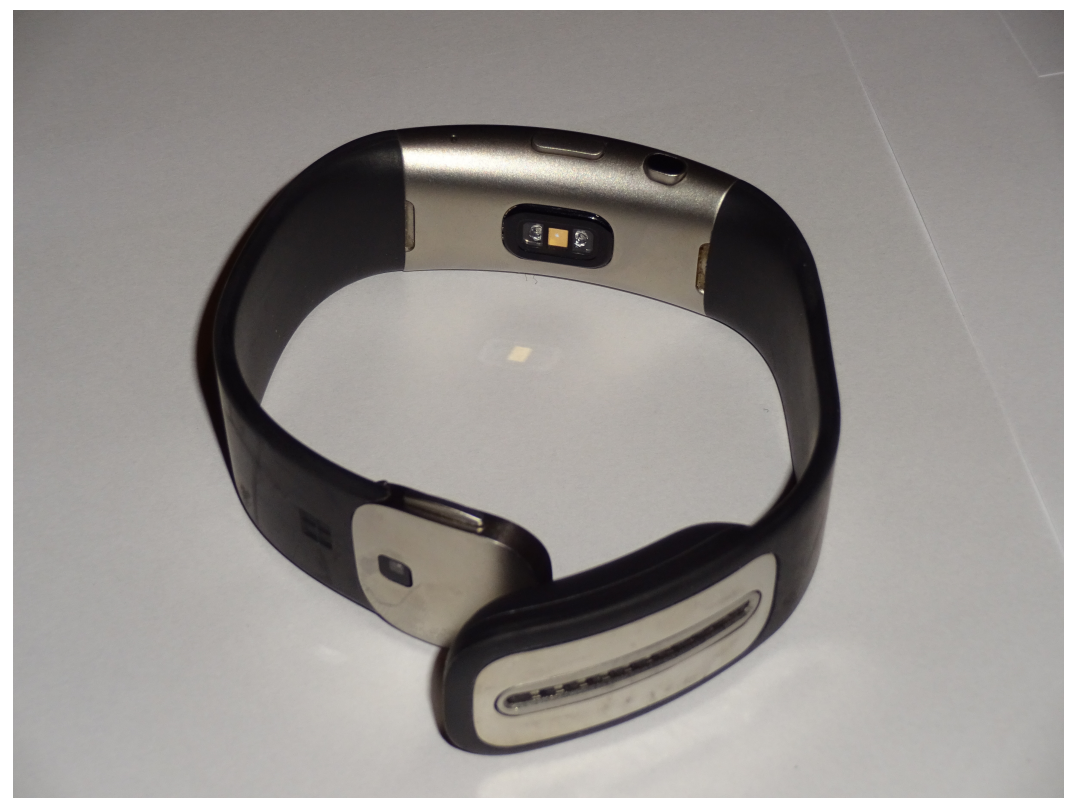

Figure 12: The smart device used in our study

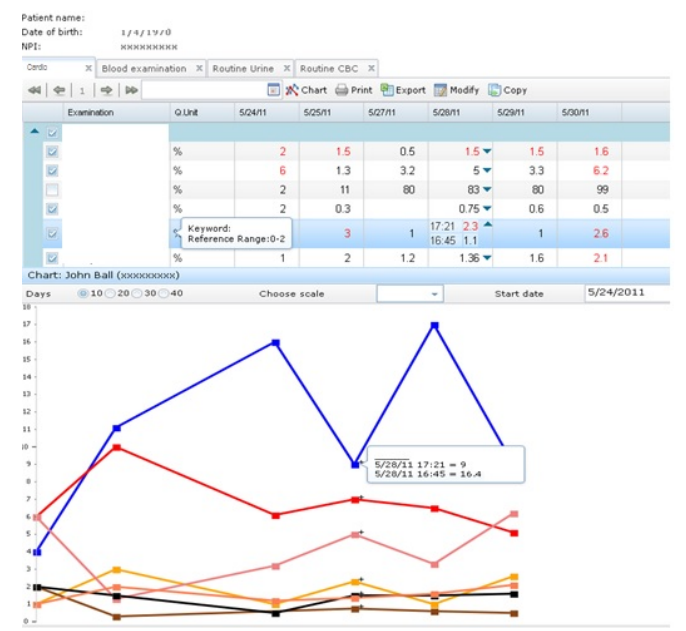

Figure 13: eHealth Smart Wearable Device Communicating via the OTI-Hub

\section{Future Work}

The key purpose of the study was to create and implement a hybrid Cloud-based prototype that provides clinical research patient data using a smart-device body- 
sensory information flow. Here, we asked ourselves whether it was possible that body-sensory smart device technology and telemedicine instrument architecture could be integrated into the classical healthcare information landscape. Now, it is clear that the answer is categorical affirmative.

Based on the results and experience gained by our research, we see that it is indeed possible to achieve the flawless interconnection of the body-sensory IoT smart device technology, the telemedicine architecture and the conventional healthcare IT landscape. Our study indicates that meaningful healthcare information exchange is possible among HL7 capable HIS and eHSDs. Another important point is that the interconnection of different platforms (UNIX, Windows) and manufacturer-specific smart devices requires special nesting software classes to handle the slightly different coding conventions and data formats. The proposed API provides a bridge between these systems and devices by specific conversions and it provides a consistent way to access all the data originating from the different parties.

The full-duplex bi-directional interoperability will constitute the next area of our research. Here, we demonstrated unidirectional simplex interoperability over the OTI-Hub. In the next phase, bi-directional functionality will be set up and evaluated. The Hub's transformation module will be relocated as an independent component outside of the Hub. This reshaped transformation module will also run in an Android app-based java class. This app itself will also transform the incoming bio-sensory dataflow into a unified data format. As a result, the OTI-Hub will be extended to interpret various incoming sensory-data acquired from different sources, as long as it is transformed into a unified format by the preloading module.

An evaluation on the interoperability of two different software manufacturer's HIS' via the OTI-Hub is planned in the next phase of our research. Telecommunication infrastructure backbones preparedness (e.g. GSM Network Bandwidth) should also be assessed in the future for securing a substantial increase in the IoT data-exchange volume.

\section{Conclusions}

As the reader, can see our OTI-Hub described above serves as a methodological basis and software solution for the interconnection of the world of IoT and conventional healthcare technology based on standards. We successfully demonstrated and tested the interoperability built on our hybrid cloud-based architectural solution with the OTI-Hub. The OTI-Hub provides an open architectural solution for solving the interoperability issue among HIS, eHSD and TI domains. Here, the data transformation into unified standard is critical for the extensibility of the presented solution. I should be added that healthcare smart device technology will generate an unprecedented amount of human body-sensory data and this will be made available in the foreseeable future. This bulk information could serve inter alia as important input for epidemic control and set new targets for pharmaceutical development. Big Data analytics methodologies foster pattern and trend analyses based on the captured body-sensory healthcare information base and offer a new 
way for crowd-sourced information handling. Our proposed hybrid cloud architecture assures the essential scalability for the OTI-Hub with its required robust transaction processing capacity. The architectural topology and systems integration solution illustrated here provides a technological solution for the integration of bi-directional international body-sensory, telemedicine and conventional healthcare data exchange. The results presented here offer some optimism, but current national healthcare data-related legal prerequisites need to be internationally harmonized for the required breakthrough. Our own OTI-Hub solution provides international eHealth data-exchange capability. Furthermore, the cloud-based eHealth interoperability solution presented here offers a framework for other application areas like the cloud-based implementation of the da Vinci Surgical System.

\section{References}

[1] Simmons S. C., Hamilton D. R., McDonald P. V. Principles of Clinical Medicine for Space Flight. Springer New York, New York, 2008, p. 163.

[2] ISO/HL7 10781:2015, HL7 Electronic Health Records-System Functional Model, Release 2, HER FM. http://www.iso.org/iso/iso_catalogue/ catalogue_tc/catalogue_detail.htm?csnumber $=57757$ (last visited on 16 September in 2016)

[3] Health Level Seven Standard Version 2.3.1 An Application Protocol for Electronic Data Exchange in Healthcare Environments, Health Level Seven International http://www.hl7.org/ (last visited on 3 March in 2017)

[4] World Development Report 2016: Digital Trends. World Bank Group, IBRD (The World Bank), Washington DC, 2016, p. 328 (354). http://documents. worldbank.org/curated/en/896971468194972881/ pdf/102725-PUB-Replacement-PUBLIC.pdf (last visited on 1 July in 2016)

[5] Definition of Big Data, Merriam-Websters Collegiate Dictionary, Web. http: //www.merriam-webster.com/dictionary/big\%20data (last visited 4 July in 2016)

[6] Zarour, K. Proposed technical architectural framework supporting heterogeneous applications in a hospital. International Journal of Electronic Healthcare, 2016, Vol. 9, Issue 1, pp. 19-41.

[7] Warren, S., Craft, R. L., Parks, R.C., Gallagher, L. K., Garcia, R. J., Funkhouser, D. R. A proposed Information Architecture for Telehealth System Interoperability. Conference: Toward an Electronic Patient Record 99, Orlando, USA, 2-6 May, 1999, pp. 1-10.

[8] Gaynor, M. G. Evaluation of Patient to Provider Oriented Telemedicine in Hospitals and Physician Practices. Muskie School Capstones, Paper 103, 2015, pp. 1-32. 
[9] Fuhrman, S. A., Lilly, C. M. ICU Telemedicine Solutions. Clinics in Chest Medicine, Elsevier, Vol. 36, Issue 3, 2015, pp. 401-407.

[10] Emery, S. Telemedicine in Hospitals Issues in Implementation. Garland Publishing, New York, 1998.

[11] Kuziemsky, C. E., Peyton, L. A framework for understanding process interoperability and health information technology. Health Policy and Technology, Vol. 5, Issue 2, 2016, pp. 196-203.

[12] Bouamrane M. M., Tao C., Sarkar I. N. Managing Interoperability and Complexity in Health Systems. Methods Inf Med, Issue 54, 2015, pp. 1-4.

[13] Wei-Li L., Kai Z., Craig L., Michael A. Cloud and Traditional Videoconferencing Technology for Telemedicine and Distance Learning. Telemedicine and e-Health, Vol. 21, Issue 5, 2016, pp. 422-426.

[14] Taylor, A., Morris G., Tieman J., Currow D., Kidd M., Carati C. Building an Architectural Model for a Telehealth Service. E-Health Telecommunication Systems and Networks, Vol. 4, No. 3, 2015, pp. 35-44.

[15] Perera C., Jayaraman P., Zaslavsky A., Christen P., Georgakopoulos D. Dynamic Configuration of Sensors Using Mobile Sensor Hub in Internet of Things Paradigm. CoRR, Vol. abs/1302.1131, 2013.

[16] Lengyel, L., Ekler P., Ujj T, Balogh T., Charaf, H. SensorHUB: An IoT Driver Framework for Supporting Sensor Networks and Data Analysis. International Journal of Distributed Sensor Networks, Hindawi Publishing Corporation, Vol. 2015, 454379, 2015.

[17] Shibuta, H., Iwata, M. Self-Timed I/O Architecture of Data-Driven Sensor Hub. Proceedings of the International Conference on Parallel and Distributed Processing Techniques and Applications, Athens, 2016, pp. 323-328.

[18] Patel H. R., Sola R. Implementations of Wireless Sensor Hub to Support Protocols Interoperability. International Journal of Research in Computer and Communication Technology, Vol. 4, Issue 4, March 2015, pp. 192-197.

[19] Gay V., Leijdekkers, P. Bringing Health and Fitness Data Together for Connected Health Care: Mobile Apps as Enablers of Interoperability. Journal of Medical Internet Research, 17(11) e260.

[20] Nijeweme-dHollosy W. O., van Velsen L., Huygens M., Hermens H. Requirements for and barriers towards interoperable eHealth technology in primary care. IEEE Internet Computing, Vol. 19, Issue 4, July-August, 2015, pp. 10-19.

[21] Kumar R. B., Goren N. D., Stark D. E., Wall D. P., Longhurst, C. A. Automated integration of continuous glucose monitor data in the electronic health records using consumer technology. Journal of the American Medical Informatics Association, Vol. 23, 2016, pp. 532-537. 
[22] Barbarito F., Pinciroli F., Mason J., Marceglia S., Mazzola K., Bonacina S. Implementing standards for the interoperability among healthcare providers in the public regionalized Healthcare Information System of the Lombardy Region. Journal of Biomedical Informations, Vol. 45, 2012, pp. 736-745.

[23] xDT, European Innovation Partnetship, European Commission, Standards, IcT and communication. https://ec.europa.eu/eip/ageing/standards/ ict-and-communication/other-ict/xdt_en (last visited on 20 January 2017)

[24] National Council for Prescription Drug Programs. http://www.ncpdp.org/ (last visited on 20 January in 2017)

[25] Organization for the Advancement of Structured Information Standards, OASIS. https://www.oasis-open.org/ (last visited on 30 January 2017)

[26] Cloud Application Management for Platforms (CAMP) TC. https:// www . oasis-open.org/committees/tc_home.php?wg_abbrev=camp (last visited on 30 January in 2017)

[27] International Health Terminology Standards Development Organisation, Systematized Nomenclature of Medicine Clinical Terms (SNOMED-CT) http: //www.ihtsdo.org/snomed-ct (last visited on 20 August in 2016)

[28] Fong B., Fong A. C. M., Li C. K. Telemedicine technologies: Information technologies in medicine and telehealth, Chichester, Wiley, 2011.

[29] Shaikh, A. The impact of SOA on a system design for a telemedicine healthcare system. Network Modeling Analysis in Health Informatics and Bioinformatics, 4:15, Springer Vienna, Vienna, 2015, pp. 1-16.

[30] Eren H., Webster J. G. The e-medicine, e-health, m-health, in Telemedicine and Telehealth Handbook, Oakville, CRC Press, 2015.

[31] Adenuga, O. A., Kekwaletswe, R. M., Coleman, A. eHealth integration and interoperability issues: towards a solution through enterprise architecture, Health Information Science and Systems, Vol. 3, Number 1, 2015, pp. 1-8.

[32] Luz, M. P., de Matos Nogueira, J. R., Cavalini, L. T., Cook T. W. Providing Full Semantic Interoperability for the Fast Healthcare Interoperability Resources Schemas with Resource Description Framework. Healthcare Informatics (ICHI), 2015 International Conference on, Dallas, 21-21 October 2015, pp. $463-466$.

[33] Garai A., Pentek I. Adaptive services with cloud architecture for telemedicine, 6th IEEE Conference on Cognitive Infocommunications, Gyor, Hungary, 19-21 October, 2015, pp. 369-374. 
[34] Adamko A., Garai A., Pentek I. Common open telemedicine hub and infrastructure with interface recommendation, 11th IEEE International Symposium on Applied Computational Intelligence and Informatics, Timisoara, Romania, 12-14 May 2016, pp. 385-390.

[35] Garai A. Methodology for assessment validation of platform migration of robust critical IT-systems, 8th International Conference on Applied Informatics, Eger, Hungary, 27-30 January 2010, pp. 445-448.

[36] Adamko A., Garai A., Pentek I. Common open telemedicine hub and interface standard recommendation, The 10th Jubilee Conference of PhD Students in Computer Science, Szeged, Hungary, 27-29 June 2016, pp. 24-25.

[37] Adamko A., Kollar L. A system model and applications for intelligent campuses: Intelligent engineering systems, 18th International Conference on Intelligent Engineering Systems, Tihany, Hungary, 3-5 July 2014, pp. 193-198.

[38] Baranyai P., Csapo A. Definition and synergies of cognitive infocommunications, Acta Polytechnica Hungarica, Vol. 9, No. 1, 2012, pp. 67-83.

[39] Adamko A., Arato M., Fazekas G., Juhasz, I. Performance evaluation of largescale data processing systems, Proceedings of the 7th International Conference on Applied Informatics, Eger, Hungary, 28-31 January 2007, Vol. 1, pp. 295301.

[40] Adamko A., Kollar L. MDA-based development of data-driven Web applications, Proceedings of the Fourth International Conference on Web Information Systems and Technologies, Funchal, Madeira, Portugal, 4-7 May 2008, Vol. 1, pp. $252-255$.

[41] ISO/IEC 7498-1:1994 Information Technology - Open Systems Interconnection - Basic Reference Model: The Basic Model (OSI-Model), International Organization for Standardization (ISO), Web. 6 June 2016. http: //www.iso.org/iso/catalogue_detail.htm?csnumber=20269 (last visited on 15 July 2016)

[42] Szabo R. et al, Framework for smart city applications based on participatory sensing, IEEE 4th International Conference on Cognitive Infocommunications, Budapest, Hungary, 2-5 December 2013, pp. 295-300.

[43] Pandi, K., Charaf, H. Mobile Resource Management Load Balancing Strategy. Acta Cybernetica, Vol. 22, Number 1, Szeged, 2015, pp. 171-181.

[44] Giachetta R., Fekete I. A Case Study of Advancing Remote Sensing Image Analysis. Acta Cybernetica, Vol. 22, Number 1, Szeged, 2015, pp. 57-79. 